\title{
¿ESCONDRIJO O BASURAL? UN APORTE DESDE EL REGISTRO ARQUEOLÓGICO VÍTREO RECUPERADO EN LA PEQUEÑA CAVIDAD DE UN BLOQUE (PROVINCIA DE SANTA CRUZ, ARGENTINA)
}

\author{
HIDING PLACE OR GARBAGE DUMP? A CONTRIBUTION FROM THE \\ VITREOUS ARCHAEOLOGICAL RECORD RECOVERED FROM THE SMALL \\ CAVITY OF A BLOCK (SANTA CRUZ PROVINCE, ARGENTINA)
}

\author{
Natalia Andrea Cirigliano ${ }^{1}$
}

\begin{abstract}
En el Valle del Cañadón Mercerá, cuenca media de la margen norte del Río Santa Cruz, se identificó un bloque con una pequeña cavidad (Oquedad 1) que contenía una elevada frecuencia de ítems vítreos. En este trabajo se presenta el registro arqueológico allí recuperado con el objetivo de evaluar, desde un abordaje tecno-morfológico, si se trató de un espacio destinado al resguardo o al descarte de materiales. Los resultados obtenidos a partir del análisis de este registro apoyan la segunda de las hipótesis, es decir, la utilización de la oquedad del bloque como espacio destinado al descarte de elementos a fines de el siglo XIX y/o principios del siglo XX. Se destaca el carácter distintivo del basural de la Oquedad 1 en el contexto de Patagonia sur y se concluye que su origen se encuentra ligado a formas más intensas de ocupar los espacios próximos al Cañadón Mercerá.
\end{abstract}

Palabras claves: sitio histórico, artefactos vítreos, uso del espacio, Patagonia sur.

In this paper we discuss an archaeological record recovered from a block with a small cavity (Oquedad 1) in the Cañadón Mercerá Valley, middle basin of the northern bank of the Santa Cruz River, which contains a high frequency of vitreous elements. Our aim was to evaluate, from a techno-morphological perspective, whether this space was used as a hiding place or as a garbage dump. Our findings support the latter hypothesis, i.e., the block cavity was used as a space to discard elements at the end of the 19th century and/or the beginning of the 20th century. This garbage dump of Oquedad 1 shows a distinctive character in the Southern Patagonia context, allowing us to conclude that it was originally shaped by more intensive ways of occupying spaces near the Cañadón Mercerá.

Key words: Historic site, vitreous artifacts, use of space, Southern Patagonia.

El área de los cañadones, localizada en la cuenca media de la margen norte del Río Santa Cruz, provincia de Santa Cruz (Argentina), registró evidencias de ocupación indígena en forma discontinua desde ca. 7700 años AP hasta momentos históricos tardíos. Estas cronologías fueron mayormente obtenidas a partir de los restos orgánicos asociados con artefactos líticos en diversos sitios, tales como Yaten Guajen 1, Yaten Guajen 12, Yaten Guajen Burbuja 1 y Mercerat 1, ubicados en tubos volcánicos, bajo reparos rocosos y en espacios al aire libre próximos a afloramientos (Cirigliano y Vommaro 2014; Franco 2008, 2013; Franco et al. 2007; 2010; 2013; 2014; 2015; entre otros).

Específicamente, en el área del Cañadón Mercerá, se identificó el sitio Mercerat 1. Se trata de un espacio al aire libre y al reparo de los vientos, próximo a un arroyo, entre bloques y afloramientos rocosos. En ese sector se dataron ocupaciones humanas en ca. 1600 años AP (Cirigliano y Vommaro 2014; Franco et al. 2014) y se reconocieron artefactos confeccionados con materias primas líticas locales y no locales, raspadores elaborados sobre vidrio de envase (Cirigliano y Vommaro 2014) y motivos rupestres grabados (Fiore y Ocampo 2009). Los artefactos de vidrio $(\mathrm{n}=2)$ fueron proporcionados por pobladores locales, y según sus testimonios, habrían sido recolectados en la superficie del sitio Mercerat 1. Estos, aunque escasos, ofrecían información sobre la presencia de grupos indígenas en ese sector, posiblemente hacia principios del siglo XX (Cirigliano y Vommaro 2014). Asimismo, el uso del área próxima al Cañadón Mercerá por grupos o familias indígenas encontró sustento en las

\footnotetext{
${ }^{1}$ Instituto Multidisciplinario de Historia y Ciencias Humanas (IMHICIHU - CONICET). Buenos Aires, Argentina. naticirigliano@hotmail.com
} 
fuentes orales (Cirigliano y Vommaro 2014; Halvorsen 2011; Gradin 1976, 2000). En estas se menciona la circulación y/o acampe de indígenas, en algunos casos de forma recurrente, a comienzos del siglo XX. En un contexto más general, cabe señalar que a fines del siglo XIX se produjo el avance de la frontera ovino ganadera en los campos de la provincia de Santa Cruz (Barbería 1995). Esto aconteció con la ocupación de las mejores tierras por parte de particulares, alcanzando a comienzos del siglo XX el sector bajo estudio (Barbería 1995; Halvorsen 2011; Reinsh com. personal 2012 y 2013). El avance criollo-europeo fue posible a partir de la circunscripción de los grupos indígenas a áreas delimitadas -reservas- y espacios marginales (p.ej., Aguerre 2000; Barbería 1995; Goñi y Nuevo Delaunay 2009; Imbelloni 1949; Nuevo Delaunay 2007; entre otros).

La evidencia material recuperada en el sitio Mercerat 1 (los dos raspadores de vidrio) junto con la información aportada por los testimonios orales impulsaron la realización de trabajos arqueológicos en el área del Valle del Cañadón Mercerá durante el año 2014. Las exploraciones y prospecciones realizadas permitieron detectar junto a un arroyo, a ca. 1,2 km del sitio Mercerat 1 , un bloque con una pequeña hendidura que contenía en su interior una alta frecuencia de ítems vítreos: fragmentos de envases y artefactos formatizados y no formatizados. Dado el contexto particular del hallazgo, este trabajo informa acerca de los materiales allí depositados desde el punto de vista tecno-morfológico. El objetivo es evaluar la funcionalidad de la Oquedad 1 (en adelante Oq 1) en el pasado. Es decir, si la Oq 1 fue un espacio destinado al almacenamiento u ocultamiento de artefactos (escondrijo) o por el contrario, al descarte intencional de elementos (basural).

\section{Antecedentes Arqueológicos y Documentales}

Variados artefactos elaborados con materias primas exóticas, especialmente vidrio de envase, fueron incorporados por las poblaciones indígenas en diversos sectores de Patagonia sur continental a partir de su interacción con poblaciones europeas y/o criollas (Belardi et al. 2013; Buscaglia 2012; Carballo Marina et al. 20002002; Casanueva et al. 2019; Castro Esnal 2014; Cirigliano y Vommaro 2014; Gómez Otero 1989-90; Goñi y Nuevo Delaunay 2009; Jackson 1991a, 1991b, 1999; Martinic y Roehrs 1991; Martinic y Prieto 1985-86; Martinic et al. 1995; Molina 1969; Nuevo Delaunay 2007, 2012; Nuevo Delaunay et al. 2017; Pallo y Borrazzo 2017; entre otros). Estos elementos fueron identificados al aire libre, al pie de paredones, bajo reparo rocoso y en diversos contextos: campamentos base, entierros y basurales, entre otros.

En particular, los basurales con elementos vítreos fueron localizados al aire libre, tanto en Chile como en Argentina. Los más cercanos a la Oq 1 fueron identificados en el área de la antigua reserva Camusu
Aike, al sur del Río Santa Cruz (Belardi et al. 2013) y en Puesto Yatel y Quintillán, en la costa sur del Lago Strobel (Nuevo Delaunay 2007, 2012). En Chile, por ejemplo, se hallaron en Cuarto Chorrillo, costa de Bahía Santiago (Jackson 1991b; Martinic y Roehrs 1991) y en el Asentamiento del Cacique Mulato, en el Valle del Río Zurdo (Jackson 1999; Martinic et al. 1995). La mayoría de las áreas destinadas al descarte se encuentra próxima a estructuras habitacionales. Algunos de los depósitos fueron generados por individuos de ascendencia indígena y otros, por acciones compartidas entre colonos e indígenas. Cabe destacar que se registraron basurales de superficies muy grandes $(>100$ $\mathrm{m}^{2}$ ), grandes (p.ej., 30,36 $\mathrm{m}^{2}$ ), medianas (p.ej., 10-12 $\mathrm{m}^{2}$ ) y pequeñas (p.ej., 5-6 m²) (Belardi et al. 2013; Martinic y Roehrs 1991; Nuevo Delaunay 2012).

A pesar de que se reconocieron ítems vítreos en diversos contextos arqueológicos, el uso de oquedades o de espacios específicos para el ocultamiento de este tipo de objetos no fue mencionado en la literatura arqueológica correspondiente a momentos históricos. No obstante, esta práctica tiene un correlato durante tiempos más tempranos de la ocupación humana en la región (p.ej., Franco et al. 2011; 2018).

Por otra parte, algunos de los documentos escritos, tales como crónicas de viajes y recopilaciones de historias de vida que datan entre mediados del siglo XVII y fines del siglo XX, advierten sobre dos prácticas indígenas del área de Patagonia que resultan de interés para los objetivos de este trabajo: (1) la limpieza y descarte de residuos; y (2) el ocultamiento u equipamiento espacial de objetos útiles.

En cuanto a la limpieza o descarte de residuos se señaló, por ejemplo, actividades de aseo y/o el uso de cueros de guanaco o de potro para el traslado de residuos a otros sectores alejados al toldo (Aguerre 2000; Musters 1911; Priegue 2007). Los testimonios obtenidos por arqueólogos o lingüistas a partir de informantes de ascendencia indígena a fines del siglo XX brindaron los datos más detallados sobre este tema. Uno de ellos es el de Paten Chapalala, miembro de la toldería tehuelche del área del Río Pinturas (NW de la provincia de Santa Cruz), quien reveló con respecto al tratamiento de la basura que “...con una rastra hecha con palos y tirada por caballo se llevaba la basura lejos del campamento. Para juntarla se usaban bolsas de cuero..." (Aguerre 2000:74). También indicó que la basura "... se sacaba todos los días, todos los días se limpiaba. Se barría el toldo, se barría alrededor de los toldos, se sacaba todo, se ponía en un cuero de guanaco o de potro, y eso se arrastraba, como un trineo y se iba a tirar lejos los huesos, todo eso se amontonaba allá lejos" (Aguerre 2000:74-75). Por otro lado, Luisa Pascual, de ascendencia Tehuelche/Araucana y habitante de las tolderías cercanas al lago Viedma (Lote 119), señaló que su "...mamá cuidaba mucho el toldo; adentro estaba barrido, lisito como este piso" (Priegue 2007:24). En vista de la información recopilada, se observa que en las fuentes 
se menciona la existencia de actividades de limpieza y en algunos casos de amontonamiento de basura, aunque no queda claro la existencia de basurales formales.

En lo que concierne a equipamiento del espacio se hace referencia a la información aportada por tres crónicas de viaje del área de Patagonia (Moreno 2007 [1879]; Musters 1911; Narborough 2007 [1694]). En estas se menciona la presencia de objetos ocultos o envueltos en cuero entre matas, rocas o sobre palos.

La primera información relevante es proporcionada por John Narborough en un contexto caracterizado por los escasos contactos entre indígenas y europeos en la costa atlántica. Este contraalmirante informó en el año 1670 para la zona de bahía San Julián que:

...dos de mis hombres vieron a dos de las gentes del país detrás de una mata en el lado este. Mis hombres fueron hacia ellos, que se marcharon y dejaron un atado de pieles bajo la mata... Mis hombres llevaron el atado a bordo para mostrármelo... Abrí el atado y eran varios sacos de pieles, con tierra roja y tierra blanca, la sustancia con que se pintan. Tenían pedernales y puntas de flechas en el atado... Había brazaletes de conchas, trozos de palos, cordeles trenzados, flechas, conchas de mejillón, conchas de armadillo y una pequeña punta de uña en un clavo a modo de punzón. Las pieles eran trozos de pieles de foca y trozo de pieles de guanaco cosidas entre sí con tripas pequeñas... Había trozos de pedernal aseguradas a la horquilla de un palo con una tripa fresca, así los sostienen para darles forma a golpes a sus puntas de flecha. Había también trozos de palos para encender fuego. Eso era todo lo que había en el atado (Narborough 2007 [1694]:112).

En este mismo sentido, y doscientos años después, se destaca la información aportada por George Musters, quien exploró el interior de la región patagónica con una partida de tehuelches septentrionales y meridionales. El viajero señaló que durante su estancia en Geylum (Norpatagonia) en 1870:

Junto á la Roca de la columna, estando de caza, descubrimos un escondrijo, perteneciente á los indios de Foyel, que contenía algo que estaba envuelto y atado con cueros; era grande la tentación que sentíamos de registrar el contenido del bulto, dejamos á éste como estaba y pusimos á Foyel sobre aviso, porque no era probable que otros fueran tan escrupulosos como nosotros. Esto confirmaba lo que había dicho Casimiro á propósito de que esos indios dejaban previsoramente bolsas de gordura y provisiones en diversos sitios, á los que esperaban volver en tiempo no remoto (Musters 1911, I:309).
Otro posible ejemplo de equipamiento lo ofrece Francisco P. Moreno en el año 1877, quien fue enviado a Patagonia sur por el Estado nacional con el objetivo de explorar el territorio y consolidar la soberanía argentina (Nacuzzi 2005 [1998]). La acción de resguardo se habría dado en el paraje tehuelche Cayick o Ayick, en proximidades del Río Shehuen o Chalía, donde María, esposa del cacique Conchingan, le había dicho a Moreno que encontraría su depósito de pinturas. A este respecto, el explorador señaló: "Lo encontramos y recojo muestras de ellas. Están envueltas en un cuero y atado éste sobre un palo..." (Moreno 2007 [1879]:207).

En suma, la información recopilada a partir del registro arqueológico y escrito advierte sobre la existencia potencial de escondrijos y basurales en Patagonia.

\section{Antecedentes Teóricos}

Según la bibliografía arqueológica, un escondrijo hace referencia a un lugar de dimensiones reducidas -p.ej., cuevas, hendiduras en rocas-, accesible y distinguible en el espacio -p.ej., cruce de arroyo- y utilizado para el acopio y ocultamiento de objetos para su uso posterior o diferido en un futuro inmediato (Binford 1978, 1979; Franco et al. 2011). Estos se encuentran en el marco de una estrategia de uso logístico del espacio, que implica la intención de regresar al sitio de manera planificada (Binford 1980). En los escondrijos se espera recuperar materias primas de óptimas calidades para la talla y/o de distintas procedencias. En este sentido, el acopio de envases o fragmentos de vidrio de tamaños adecuados y de excelente calidad para la talla podría ser esperable en el área, ya que entre las materias primas líticas locales solo la calcedonia la iguala en aptitud, y de esta roca solo se registraron nódulos de tamaños pequeños, de disponibilidad escasa y distribución impredecible (Franco y Cirigliano 2009; Franco et al. 2015). También se podrían reconocer artefactos de grandes dimensiones y/o en las primeras etapas de manufactura, instrumentos específicos enteros -p.ej., bifaces, puntas, raspadoreso con potencialidad de uso y/o alta frecuencia de instrumentos en diferentes etapas de manufactura -p.ej., bifaces- (Ainis et al. 2017; Amick 2004; Bement et al. 1991; Erlandson et al. 2013; Franco et al. 2011; 2018; Grange 1964; Kornfeld et al. 1990, Rennie y Davis 2016; Oliva et al. 1991).

Los basurales, en cambio, se conforman de elementos cuya vida útil ha concluido (Schiffer 1972). Estos se pueden clasificar en basura primaria o secundaria. La primera refiere a ítems descartados en el lugar de uso y la segunda, a elementos transportados y desechados en otra ubicación (Schiffer 1972). A su vez, los basurales se pueden dividir en dos tipos: los basurales principales o generalizados tendrían tamaños grandes y una amplia variedad de desechos, mientras que los basurales secundarios o específicos se caracterizarían por presentar 
tamaños pequeños y baja diversidad de residuos (Borrero y Yacobaccio 1989). En líneas generarles, en caso de basural se espera encontrar materias primas de tamaños no óptimos para la confección de artefactos, desechos de talla o instrumentos de pequeñas dimensiones, distintas etapas del proceso de manufactura de instrumentos, artefactos formatizados fragmentados, reactivados o mantenidos y/o con ángulos embotados (Borrero y Yacobaccio 1989; Jackson 1991b, 1999; Martinic y Roehrs 1991; Nuevo Dealunay 2007; Schiffer 1972; entre otros).

En resumen, y en vista de los antecedentes teóricos previamente señalados, las expectativas en caso de escondrijo involucrarían la posibilidad de reconocer ítems con potencialidad de uso. Por el contrario, en el caso de basural, se esperaría recuperar elementos sin ninguna utilidad. En el primero de los casos, la oquedad bajo estudio funcionaría como un lugar de acopio y resguardo de materiales, mientras que en el segundo, como un locus seleccionado para el descarte final de elementos inservibles.

Es significativo señalar que este trabajo se enmarca en la "Arqueología de Momentos Históricos" (sensu Goñi y Madrid 1998:71) y acuerda con la idea de que el registro arqueológico tiene la capacidad de dar explicaciones propias acerca el pasado reciente (Goñi y Madrid 1998; Goñi y Nuevo Delaunay 2009; entre otros).

\section{La Oquedad 1}

La Oq 1 se encuentra localizada en el Valle del Cañadón Mercerá, margen norte del Río Santa Cruz, en un paisaje caracterizado por estructuras mesetiformes de composición basáltica (Oliva et al. 2001) y a ca. $30 \mathrm{~m}$ de un afloramiento rocoso (Figura 1). En sus proximidades se encuentra el Cañadón Mercerá, un curso de régimen efímero que sólo registra agua en épocas de lluvias (Panza et al. 2005). A su vez, la Oq 1 se ubica junto a un arroyo que continúa su curso hacia el sur, labrando su cauce en las inmediaciones del sitio Mercerat 1 (ver arriba).

La hendidura del bloque (Figura 1), detectada durante los trabajos de campo en el año 2014, con un volumen o espacio aproximado de $0,092 \mathrm{~m}^{3}$, contenía en su interior una alta frecuencia de ítems vítreos $(\mathrm{n}=2.436)$. La misma fue cepillada y vaciada en su totalidad, obteniendo únicamente el material arqueológico aquí bajo estudio. Entre los elementos de vidrio se reconocieron fragmentos de envases, desechos de talla y artefactos formatizados. En superficie y en proximidades a la Oq 1 se recuperaron artefactos vítreos y líticos, fragmentos de vidrio, objetos de metal y restos óseos de caballo. Los ítems vítreos allí recolectados alcanzan un total de 381, una cantidad bastante menor a aquellos identificados en el interior de la oquedad.
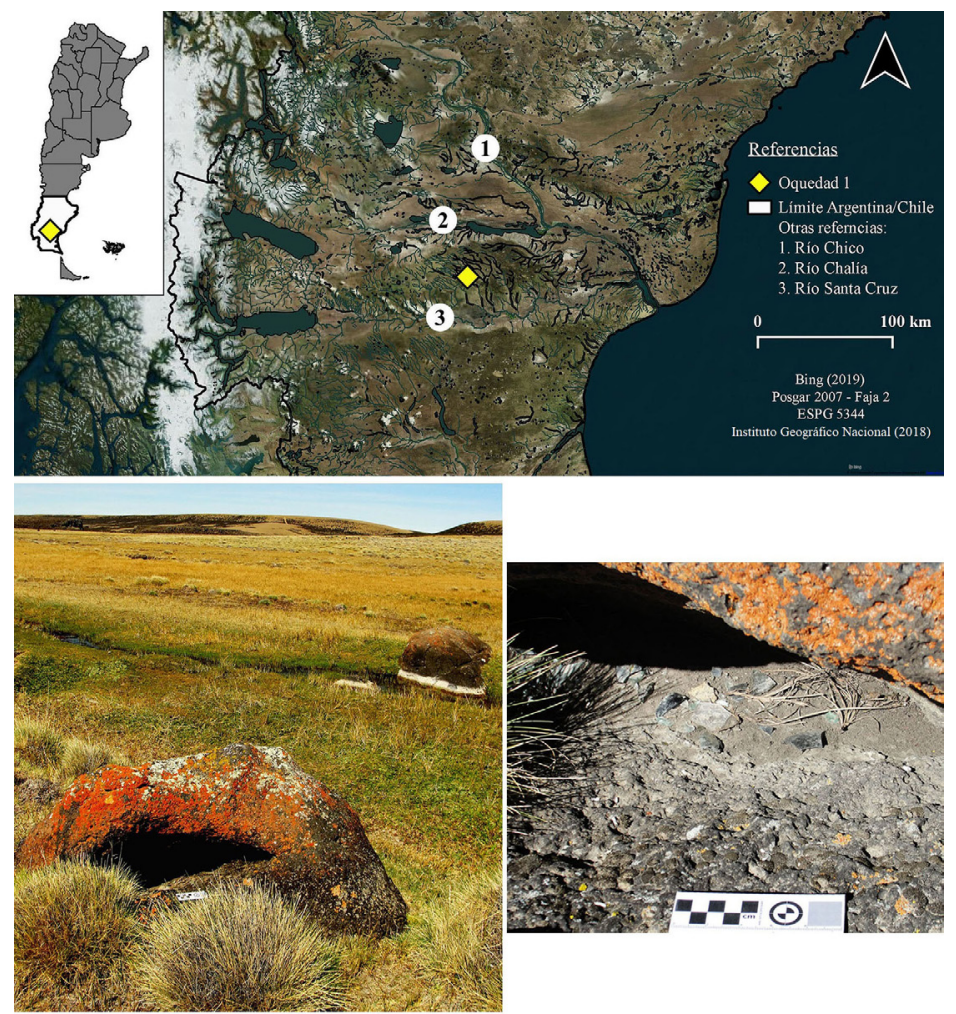

Figura 1. Localización y vista de la Oquedad 1.

Location and view of the Oquedad 1. 


\section{Metodología}

El análisis de los artefactos vítreos siguió los lineamientos propuestos por Aschero (1975, 1983). En todos los casos, entre los desechos de talla se consignó talón (presente/ausente) y tipo de extracción (lasca/ hoja). Un análisis más específico se realizó sobre un $25 \%$ de los ejemplares enteros con el objetivo de tener una primera aproximación a la variabilidad de los tipos presentes. Los tamaños de los artefactos (formatizados y no formatizados) y fragmentos de envases fueron considerados como muy pequeños $(0,1-20 \mathrm{~mm})$, pequeños (20,1 - $40 \mathrm{~mm}$ ), medianos pequeños (40,1 - 60 $\mathrm{mm})$, medianos grandes $(60,1-80 \mathrm{~mm})$, grandes $(80,1$ - $100 \mathrm{~mm}$ ) y muy grandes (>100,1 cm), a partir de la metodología de grilla (intervalos de $5 \mathrm{~mm}$ ) propuesta por Franco (2002). Para el caso de los artefactos formatizados, los tamaños fueron también medidos con calibre a partir de su eje morfológico (sensu Aschero 1983).

Asimismo, se buscó evaluar si los fragmentos de vidrio podían o no ser apropiados para confeccionar artefactos formatizados. Para esto se consideraron: (1) las dimensiones de los fragmentos de botellas seleccionados por un grupo de quillangueras tehuelches de la provincia de Santa Cruz ${ }^{1}$ para la elaboración de raspadores (Gómez Otero 1987; 1996-97) y (2) el tamaño de los raspadores hallados en el interior de la oquedad bajo estudio.

Los elementos vítreos registraron diversas coloraciones y en algunos casos distintas tonalidades. Esto podría deberse tanto a la existencia de diferentes envases como a la variabilidad de espesores hallada en un mismo envase o entre artefactos tallados. No obstante, para los objetivos de este trabajo y al fin de disminuir el sesgo, fueron agrupados en cuatro grandes clases de colores: transparente, aqua (celeste), ámbar verdoso y verde. Resta mencionar que se reconocieron algunos fragmentos de vidrio y artefactos (formatizados y no formatizados) patinados o tornasolados, mientras que otros no registraron esta característica. Es importante destacar que este rasgo no puede ser considerado como evidencia de que algunos elementos son más antiguos que otros (Ortiz Castro 2007). La pátina en vidrio se puede generar por diversos factores como el enterramiento, la presencia de agua, la exposición al calor y la composición química de material, entre otros (Lorrain 1968; Sironi 2010).

\section{Resultados}

\section{Los materiales vítreos de la Oquedad 1}

La pequeña cavidad del bloque alojaba en su interior 2.436 ítems vítreos, entre los que se reconocieron fragmentos de envases $(9,81 \%)$, desechos de talla con y sin talón $(89,00 \%)$ y artefactos formatizados $(1,19 \%)$ (Tabla 1 y Figura 2).

Tabla 1. Conjunto vítreo.

\begin{tabular}{ccccc}
\multicolumn{5}{c}{ vitreous assemblage. } \\
\hline Color & $\begin{array}{c}\text { Fragmento } \\
\text { de envase }\end{array}$ & $\begin{array}{c}\text { Desecho } \\
\text { de talla }\end{array}$ & Instrumento & Total \\
\hline Transparente & 16 & 625 & 1 & 642 \\
Aqua & 18 & 970 & 6 & 994 \\
Ámbar & 67 & 47 & 5 & 119 \\
verdoso & 138 & 526 & 17 & 681 \\
Verde & 239 & 2.168 & 29 & 2.436 \\
\hline Total & & & & \\
\hline
\end{tabular}

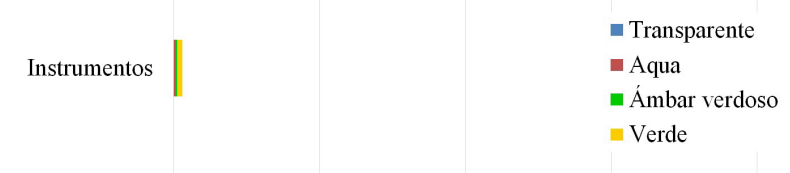

Lascas con talón

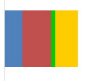

Lascas sin talón

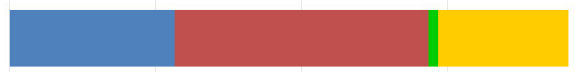

Fragmentos

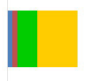

0

500

1000

1500

2000

Frecuencia

Figura 2. Conjunto arqueológico vítreo y su coloración.

Archaeological vitreous assemblages and it coloration. 


\section{Fragmentos de envases}

Los fragmentos de vidrio $(n=239)$ encuentran su origen en diversos envases. Entre estos se reconocieron fragmentos de cuerpo, pico, cuello, hombro, base e indeterminado, siendo en su mayoría clasificados como segmentos de cuerpo. Los ejemplares son de coloración transparente (6,70\%), aqua (7,53\%), ámbar verdoso $(28,03 \%)$ y verde $(57,74 \%)$. Los valores de tamaños entre los fragmentos se distribuyen entre 5,1 y $75 \mathrm{~mm}$; es decir, entre muy pequeños y medianos grandes.

\section{Artefactos no formatizados}

Entre los desechos de talla $(\mathrm{n}=2.168)$ se registraron en orden de importancia lascas de coloración aqua $(44,74 \%)$, transparente $(28,83 \%)$, verde en variadas tonalidades $(24,26 \%)$ y ámbar verdoso $(2,17 \%)$ (Figura 3). De estos, solo el $10,60 \%(n=230)$ de la muestra se encontró entero y el 11,62\% ( $\mathrm{n}=252)$ registró talón. El resto de los desechos se conforma por lascas de tamaños reducidos y sin talón (estallados/astillados y fragmentos no proximales). Si se consideran únicamente los ejemplares con talón, se identifican en orden de importancia aquellas lascas de coloración aqua $(39,68 \%)$, verde $(30,95 \%)$, transparente $(23,81 \%)$ y ambar-verdoso $(5,56 \%)$.

Las dimensiones de los desechos de talla enteros se encuentran comprendidas entre los 0,1 y $35 \mathrm{~mm}$ (tamaños muy pequeños y pequeños), aunque los tamaños muy pequeños $(\leq 20 \mathrm{~mm})$ son predominantes en la muestra $(98,26 \%)$. El análisis del $25 \%$ de los desechos enteros seleccionados al azar $(\mathrm{n}=58)$ permitió identificar lascas de reactivación directa $(\mathrm{n}=28 ; 48,28 \%)$, de arista $(\mathrm{n}=17 ; 29,31 \%)$, planas $(\mathrm{n}=6 ; 10,34 \%)$, angulares $(\mathrm{n}=4$; $6,90 \%)$ e indiferenciadas $(n=3 ; 5,17 \%)$. Los talones representados son en orden de importancia filiformes $(n=33 ; 56,90 \%)$, puntiformes $(n=15 ; 25,87 \%)$, lisos $(\mathrm{n}=8 ; 13,79 \%)$, diedros $(\mathrm{n}=1 ; 1,72 \%)$ y facetados $(\mathrm{n}=1$; $1,72 \%)$.

\section{Artefactos formatizados}

Se obtuvieron 29 artefactos formatizados sobre fragmentos de vidrio de envase de coloración verde (58,62\%), aqua (20,69\%), ámbar-verdoso $(17,24 \%)$ y transparente $(3,45 \%)$. Entre los grupos tipológicos se reconocieron únicamente raspadores $(n=28)$, con la excepción de un ejemplar que fue asignado como instrumento no diferenciado por fractura (Figura 4). La formatización de los filos se realizó en mayor medida sobre la sección correspondiente al cuerpo de la botella y, en menor medida, sobre sus hombros y hombro-cuello. En algunas pocas ocasiones el sector del envase utilizado no pudo ser determinado. Cuando se utilizó el cuerpo, los retoques se efectuaron sobre fragmentos transversales al eje longitudinal de la botella, lo que permitía aprovechar su curvatura original. Cabe señalar que el $89,66 \%$ de los instrumentos registró algún tipo de fractura.

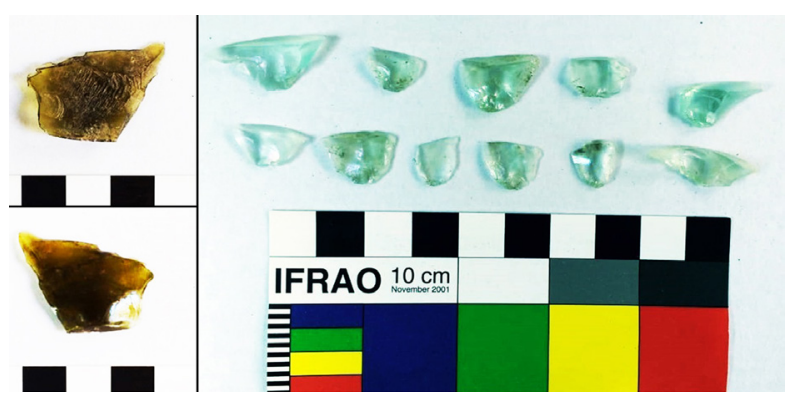

Figura 3. Lascas de vidrio.

Glass flakes.

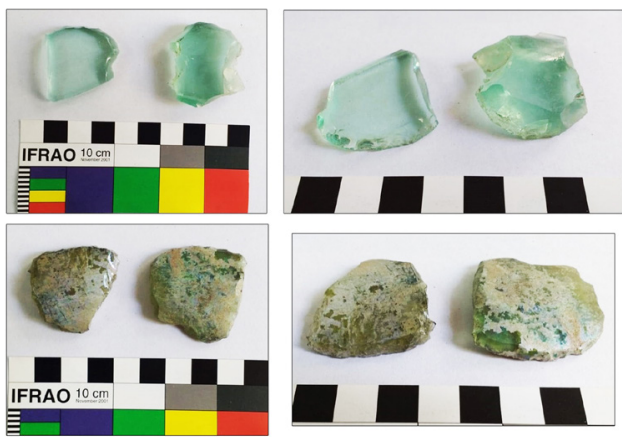

Figura 4. Raspadores recuperados en la Oquedad 1. Derecha: Detalle de los filos retocados.

Scrapers recovered at Oquedad 1. Right: Detail of the retouched edges. 
Los raspadores $(\mathrm{n}=28)$ fueron determinados como de filo frontal $(n=10 ; 35,72 \%)$, fronto-lateral $(n=3$; $10,71 \%)$, fronto- bilateral $(\mathrm{n}=1 ; 3,57 \%)$, perimetral $(\mathrm{n}=2 ; 7,14 \%)$ y no diferenciados por fractura $(\mathrm{n}=12$; $42,86 \%$ ). Estos fueron confeccionados por medio de retoques o de retoques y microretoques realizados de forma unifacial directa, siendo de profundidad y extensión marginal. Dada la alta fragmentación de las piezas, la extensión del filo debe ser considerada con precaución, pudiendo reconocer en estas filos cortos $(n=3), \operatorname{largos}(n=7)$, extendidos $(n=4)$, perimetrales $(n=2)$ e indiferenciados por fractura $(n=12)$.

Los ángulos de los filos de raspador fueron medidos en todos los casos ( $\mathrm{n}=28$ ) (Figura 5; Ángulos de filos). Los ángulos iniciales fueron proyectados entre los $50^{\circ}$ y $65^{\circ}$, mientras que los medidos entre los $60^{\circ}$ y $135^{\circ}$. Cabe destacar que los valores altos de desgaste solo se encontraron en una pequeña porción del filo que registraba mayor abrasión. El 96,43\% de la muestra presenta filos que pueden ser considerados como embotados (ángulo $\geq 70^{\circ}$ ). Los ángulos de desgaste son muy variables (entre $15^{\circ}$ y $75^{\circ}$ ) y el valor con mayor representación en la muestra es de $40^{\circ}(21,43 \%)$. El $75 \%$ de los raspadores registró evidencia de reactivación de filos.

Con relación a las dimensiones de los raspadores, cuya medida fue registrada a partir de los sectores que no presentaban fracturas, se estableció la longitud, el ancho y el espesor de las piezas. El largo de los instrumentos se encontró comprendido entre los $25 \mathrm{~mm}$ y $43 \mathrm{~mm}(\mathrm{n}=20)$, el ancho entre los 29 y $38 \mathrm{~mm}(\mathrm{n}=8)$ y el espesor entre los 2,5 y $10 \mathrm{~mm}(\mathrm{n}=28)$ (Figura 5, Dimensiones). La media para el largo fue registrada en $32,45 \mathrm{~mm}$, para el ancho en $33,81 \mathrm{~mm}$ y para el espesor en $4,39 \mathrm{~mm}$. De estos datos se desprende que la longitud es lo que más varía entre los raspadores, sugiriendo muy posiblemente una mayor reducción del largo que del ancho de las piezas. Sin embargo, cabe señalar que la anchura se encuentra mayormente afectada por las fracturas. Por otra parte, los espesores registraron poca variación en sus tamaños. Esto se fundamenta en el uso de materias primas cuya morfología se encuentra predeterminada, condicionando los espesores de las piezas a la variabilidad original preexistente (envases). Cabe señalar que los instrumentos enteros registran tamaños pequeños, entre 30,1 y $40 \mathrm{~mm}$.

El instrumento no diferenciado se confeccionó mediante retoques localizados en forma unifacial directa, de profundidad y extensión marginal. Su ángulo inicial fue estimado en $65^{\circ}$ y el medido en $85^{\circ}$. La forma secundaria del filo no pudo ser determinada. Posiblemente, dado el ángulo y el contexto de hallazgo, se pueda tratar de un fragmento de raspador.

Son escasos los ítems del conjunto vítreo de la Oq 1 que se pueden utilizar para estimar cronologías. Entre los fragmentos de envases de coloración más
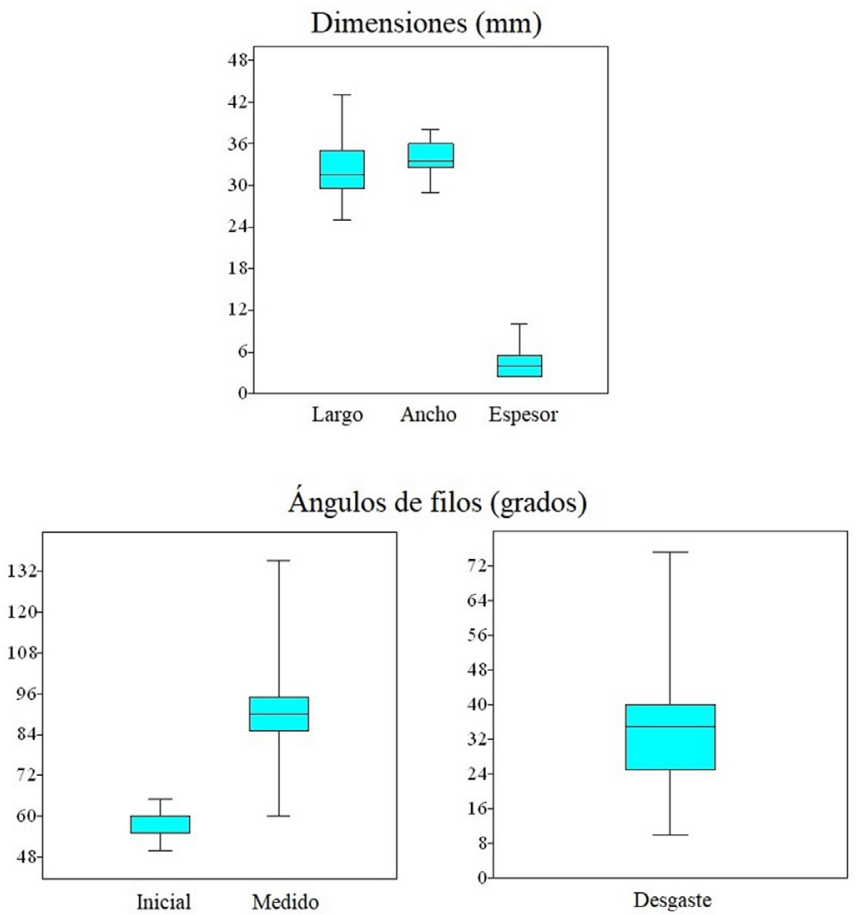

Figura 5. Gráfico de cajas (PAST, Hammer 2017). Dimensiones de los raspadores y ángulos de los filos retocados.

Boxplots (PAST, Hammer 2017). Scrapers sizes and retouched edge angles. 
oscura se reconocen picos aplicados con pinzas cuya producción se desarrolló durante el siglo XIX y una base cuadrada con aristas redondeadas que podría corresponder a un ejemplar soplado a molde de fines del siglo XIX (Virginia Pineau, com. personal 2015 y 2020). Asimismo, se reconoció una pieza grabada con ácido. Esta técnica fue principalmente utilizada a fines del siglo XIX y principios del siglo XX (Lindsey 2020). Por otra parte, los colores más diagnósticos del conjunto vítreo son consistentes con la cronología aquí indicada. El color aqua se vuelve escaso a partir de 1920 (Ortiz Castro 2007), mientras que el color transparente fue de uso recurrente durante las primeras décadas del siglo XX (Kendrick 1968; Ortiz Castro 2007). En vista de que algunos de los elementos transparentes registran un tinte rosado se considera probable que la manufactura de esos envases se haya desarrollado entre 1880 y 1916 (Ortiz Castro 2007).

\section{La potencialidad de uso de los materiales recuperados en la Oquedad 1}

Luego de describir las características tecnomorfológicas y métricas de los ítems vítreos alojados en la cavidad del bloque, es necesario discutir cuáles son las potencialidades de uso que estos presentan. En este sentido, para conocer si los fragmentos de vidrio o los desechos de talla alojados en la $\mathrm{Oq} 1$ son útiles a los fines de confeccionar raspadores, o para saber si las lascas cuentan con los tamaños apropiados para hacer uso de sus filos naturales, se compararon los tamaños de los tres subconjuntos arqueológicos -fragmentos de envases, desechos de talla enteros y artefactos formatizados- (Figura 6). Para esto, como ya se mencionó, se utilizó la grilla de tamaños elaborada por Franco (2002). Asimismo, y a fines comparativos, se consideraron los tamaños de los fragmentos de envase seleccionados por las quillangueras tehuelches de la provincia de Santa Cruz para confeccionar raspadores de vidrio (Gómez Otero 1987, 1996-97). Cabe mencionar que las quillangueras señalaban la necesidad de elaborar raspadores de gran tamaño (70 ó $50 \mathrm{~mm}$ de largo por $40 \mathrm{~mm}$ de ancho; es decir entre aproximadamente 2.000 y $2.800 \mathrm{~mm}^{2}$ ) debido al rápido embotamiento de los filos, lo que acarreaba la necesidad de reavivarlos continuamente (Gómez Otero 1987, 1996-97). En relación con lo anterior, cabe también destacar la recuperación de un raspador entero de gran tamaño en uno de los basurales del sitio Cuarto Chorrillo, costa de Bahía Santiago, Estrecho de Magallanes. Sus medidas son $100 \mathrm{~mm}$ de longitud por $58 \mathrm{~mm}$ de ancho $\left(5.800 \mathrm{~mm}^{2}\right)$, aunque confeccionado sobre vidrio plano posiblemente de ventana (Jackson 1991b).

En la Figura 6 se observa que el conjunto de desechos de talla de condición entera registra tamaños muy pequeños y pequeños $(\leq 35 \mathrm{~mm})$, posiblemente resultado de la producción y el mantenimiento de los

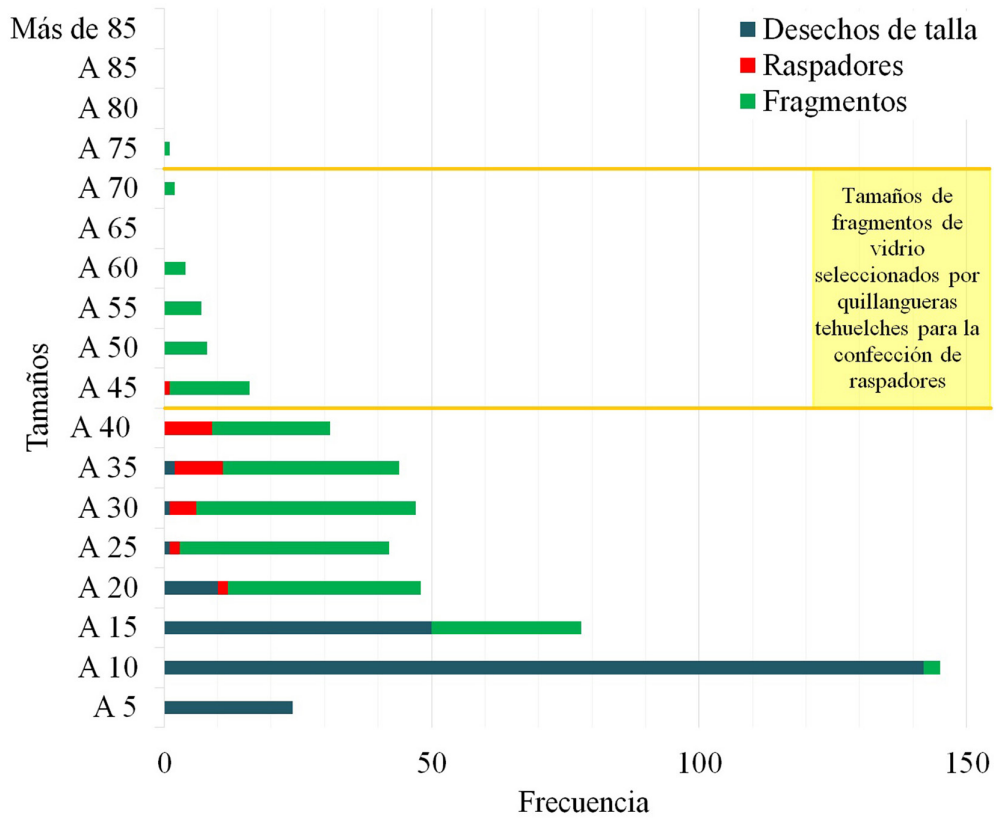

Figura 6. Comparación de tamaños y frecuencias.

Frequency and sizes comparison. 
filos de raspadores. Los tipos de lascas representados (planas, de arista, angulares, de reactivación directa) y la variabilidad de talones apuntan también en este sentido. Las lascas mayores a $20 \mathrm{~mm}$ y potencialmente utilizables por sus filos cortantes son muy escasas $(n=4$; $1,74 \%)$.

La sub-muestra de raspadores (enteros y fragmentados) presentó tamaños $\leq$ a $45 \mathrm{~mm}$ (Figura 6). Dada la gran intensidad de uso que se observa entre los raspadores (más de un filo retocado, filos embotados, altos ángulos de desgaste, evidencia de reactivación y fractura), es esperable que al comienzo de su vida útil presentaran tamaños mayores a los aquí registrados. Por otra parte, si se consideran las dimensiones observadas por Gómez Otero (1987, 1996-97) sobre la selección de trozos de envases por parte de las quillangueras para la confección de raspadores y el tamaño del raspador más grande $(45 \mathrm{~mm})$, aunque fracturado de la Oq 1 , de todos los fragmentos de vidrio relevados, solo una pequeña cantidad ( $\mathrm{n}=14 ; 5,86 \%)$ podría por su tamaño $(\geq 55 \mathrm{~mm})$ ser utilizada como materia prima para la confección de esta clase de instrumento (Figura 6). En relación con lo anterior, solo resta mencionar que el trozo de envase más grande $(75 \mathrm{~mm})$ de la sub-muestra de fragmentos de vidrio corresponde a la sección del pico y el cuello de una botella, parte generalmente no utilizada como forma base para la confección de raspadores. Por último, cabe señalar que el área (largo por ancho) de los raspadores enteros y fragmentados es en todos los casos menor (mínimo 264 $\mathrm{mm}^{2}$ y máximo $1.634 \mathrm{~mm}^{2}$ ) que la de aquellos trozos de envases seleccionados por las quillangueras $\left(2.000 \mathrm{~mm}^{2}\right.$ y $2.800 \mathrm{~mm}^{2}$ ), lo que podría indicar, bajo el supuesto de la selección de fragmentos de tamaños semejantes, una alta y mediana-alta intensidad de uso de los raspadores de la Oq 1.

\section{Discusión}

En un primeromomento, en base alas características del hallazgo -materiales depositados de forma oculta en una hendidura de pequeñas dimensiones- y su localización -inmediatamente próxima a un arroyo, accesible y distinguible-, surgió la necesidad de evaluar la potencialidad de la Oq 1 como un espacio destinado al resguardo de objetos. No obstante, y pese a que se identificó vidrio, materia prima alóctona y de excelente calidad para la talla, las características observadas en los materiales recuperados no cumplen con lo esperado para un escondrijo (ver arriba). Por el contrario, estas se asemejan a aquellas de los conjuntos recobrados en los basurales de Patagonia sur. En este sentido, entre los raspadores, se reconocieron piezas enteras y fragmentadas, sub-grupos y tamaños variables, y filos reactivados y/o embotados (p.ej., Jackson 1991b, 1999; Martinic y Roehrs 1991; Nuevo Delaunay 2007) que apoyan en conjunto su utilización. Asimismo, se identificaron trozos de envases no óptimos para la manufactura de raspadores, ya sea por sus secciones -picos y cuellos- (p.ej., Jackson 1999; Goñi y Nuevo Delaunay 2009; Nuevo Delaunay 2007) o por sus tamaños reducidos.

Se destaca la importancia de la información aportada por las artesanas de ascendencia tehuelche (Gómez Otero 1987, 1996-97) a partir de las cuales se elaboraron expectativas acerca del tamaño potencial de los raspadores y de los fragmentos de envase seleccionados para su manufactura. Estas, al ser cotejadas con las características del conjunto vítreo recuperado en la Oq 1 (p.ej.,raspadores más pequeños de lo esperado, fragmentos de envases de tamaños pequeños y/o secciones no óptimas para la confección de raspadores) permitió reforzar la idea del uso de la cavidad del bloque como basural. La existencia de raspadores de dimensiones menores a los tamaños de los fragmentos seleccionados por las quillangueras sugiere una considerable intensidad de uso (ver también Jackson 1991a para el sitio Dinamarquero, Chile). Esto se ve apoyado por los altos ángulos de desgaste y las evidencias de reactivación de filos en instrumentos, entre otros indicadores ya mencionados.

La coexistencia de fragmentos de vidrio, desechos de talla e instrumentos de la misma coloración consignados en la oquedad muestra la realización de distintas etapas del proceso de manufactura de raspadores, lo que también ha sido señalado para otros basurales de Patagonia (p.ej., Nuevo Delaunay 2007). Es posible que la alta variabilidad de los trozos de envases (pico, cuello, cuerpo, base) y la diversidad de los tipos de lascas y talones representados en la Oq 1 puedan dar cuenta de estadios iniciales e intermedios de talla en esta materia prima. Asimismo, la alta frecuencia de desechos de talla de tamaños muy pequeños $(\leq 20 \mathrm{~mm}$ ), las clases de talón (p.ej., filiformes y puntiformes) y los tipos de lascas identificadas (p.ej., de reactivación directa, de arista), apuntarían mayormente a la realización de estadios finales de talla y mantenimiento de filos.

En vista de que los elementos recuperados en la oquedad no resultan apropiados desde el punto de vista económico para continuar siendo utilizados, es que se asume que esta podría haber funcionado como basural. El depósito de la Oq 1 se encontraría conformado por ítems transportados desde el lugar de talla y/o uso hacia el interior de la oquedad (basura secundaria sensu Schiffer 1972). Asimismo, los tamaños pequeños de los elementos obtenidos y la baja diversidad del conjunto apuntarían a un basural de tipo secundario o específico (sensu Borrero y Yacobaccio 1989). Es significativo destacar que el gran número de desechos de talla, por ejemplo, de coloración transparente o aqua, con relación al de los raspadores (Tabla 1) sugeriría la existencia de más artefactos formatizados, los cuales 
no fueron depositados en la oquedad con el resto de los materiales.

A pesar de las semejanzas detectadas entre los materiales vítreos de los basurales de Patagonia sur, se reconocen diferencias en torno a los tamaños o localización de los loci. El lugar de depositación de los materiales vítreos, en este caso al interior de una pequeña cavidad de un bloque $\left(0,092 \mathrm{~m}^{3}\right)$, contrasta con la del resto de los basurales de momentos históricos. Estos últimos, de superficie más extensa, solo fueron reconocidos al aire libre (Belardi et al. 2013; Nuevo Delaunay 2007; Martinic et al. 1995; entre otros). En este contexto se destaca el carácter particular de la Oq 1 , ya que el uso de cavidades muy pequeñas para descartar elementos inservibles no ha sido por el momento reseñado por las investigaciones arqueológicas o las fuentes históricas. Es significativo advertir que esta forma de gestionar la basura podría tener implicancias en la detección de los sitios arqueológicos de momentos históricos, ya que tendería a reducir la visibilidad de los materiales vítreos disponibles en superficie.

Se considera importante indagar con mayor profundidad en los comportamientos que pudieron generar el registro arqueológico aquí bajo estudio. En este sentido, es posible que las actividades de limpieza desarrolladas en el pasado hayan generado este espacio formal de descarte. Este tipo de práctica podrían relacionarse con la ocupación prolongada, la re-ocupación, o el re/uso de espacios próximos a la oquedad, donde los fragmentos y desechos de talla de vidrios, filosos y dispersos, podrían haber sido una molestia o un estorbo para los ocupantes (O'Connell 1995; Hayden y Cannon 1983). Incluso, el uso de cueros para envolver basura, tal y como indican las fuentes documentales (Aguerre 2000), podría explicar la alta cantidad de desechos de talla y fragmentos de envase de tamaños pequeños y muy pequeños en la Oq 1 .

Como ya se mencionó, la Oq 1 constituye solo un espacio acotado dentro del sitio Pozo de Campos 1. En este sentido, otro factor a considerar es la menor frecuencia de fragmentos y artefactos vítreos recuperados en la superficie del sitio $(\mathrm{n}=381 ; 13,53 \%)$ con relación a los reconocidos en la pequeña cavidad del bloque $(n=2.436 ; 86,47 \%)$, hecho que podría reforzar la existencia de actividades de limpieza en proximidades a la Oq 1. Cabe destacar que las relaciones entre la Oq 1 y el resto de los materiales identificados en los sitios Pozo de Campos 1 y Mercerat 1 (ca. 1,2 km en línea recta hacia el sur) serán analizadas con mayor profundidad en futuras investigaciones y a la luz de los resultados aquí obtenidos.

Por último, es importante recordar que a fines del siglo XIX se inició el avance criollo-europeo y ganadero en los campos de Santa Cruz, alcanzando a comienzos del siglo XX el sector bajo estudio. Los ítems diagnósticos de los envases de vidrio han sido asignados a momentos de producción correspondientes al siglo XIX y principios del siglo XX. La Oq 1 podría haber sido utilizada tanto en el lapso señalado como de forma más circunscripta en el tiempo, posiblemente entre fines del siglo XIX y/o principios del $\mathrm{XX}$, cuando la presencia de las estancias hizo que esta materia prima fuera más accesible en el área. En este último caso, la diversidad cronológica daría cuenta del tiempo transcurrido entre la manufactura de los envases y su utilización como materia prima para la talla de artefactos en este sector del espacio.

\section{Conclusión}

Dadas las condiciones particulares del registro arqueológico hallado en la Oquedad 1; vale decir, una gran cantidad de materiales vítreos alojados en una pequeña cavidad de un bloque, es que se buscó indagar acerca de su potencialidad: ¿Fueron estos ítems ocultos como parte de una estrategia de equipamiento de sitio? $\mathrm{O}$, por el contrario, ¿éstos solo fueron descartados como basura secundaria?

Los datos aquí analizados apuntan al uso de la oquedad como basural. El lugar de depositación y su carácter circunscripto (pequeña cavidad) invitan a pensar que el descarte de materiales en ese lugar específico tenga que ver con la limpieza del espacio, probablemente debido al carácter cortante de los elementos de vidrio. Las actividades de limpieza pueden ser asociadas al uso prolongado y/o el re-uso del sector del Valle del Cañadón Mercerá por grupos indígenas. Esto es coincidente con lo señalado por las fuentes orales (ver arriba), y es evidencia de la estructuración del espacio durante momentos históricos tardíos.

En suma, este trabajo reveló el carácter especial de la Oquedad 1, agregando nueva evidencia sobre una forma particular de ocupar el espacio y gestionar los residuos por parte de grupos indígenas en la porción central de la provincia de Santa Cruz, Argentina. Asimismo, esta investigación permitió indagar en los comportamientos que pudieron haber generado un registro arqueológico de baja visibilidad. En este sentido, es posible que las estrategias de limpieza efectuadas en el pasado tengan implicancias en el reconocimiento de los sitios arqueológicos de momentos históricos. En este caso particular, la distribución acotada y el carácter oculto de los materiales de la Oq 1 lo vuelve un registro arqueológico de escasa visibilidad y de difícil detección en el área bajo estudio.

Agradecimientos: Los trabajos fueron financiados con los proyectos PIP (CONICET) 0447, PICT 20152038 (ANPCyT) y UBACyT (Universidad de Buenos Aries) 20020130100664BA y 20020170100663BA, dirigidos por Nora Franco. El trabajo forma parte de una beca de Doctorado CONICET (2010-2015; Cirigliano 2016). Se agradece a los pobladores de la provincia de Santa Cruz, especialmente a Mónica Reinsh (Estancia 
La Marta) y Rubén Hudson (Estancia Yaten Guajen). A Cloromiro Campos, Carlos Castro y puesteros de Ea. La Marta. Quiero agradecer principalmente a mi compañero de equipo y amigo Agustín Acevedo, quien colaboró intensamente y codo a codo en los trabajos de campo en Pozo de Campos 1, lugar donde se reconoció la oquedad aquí investigada. A Carlos Pesqueira (que ya no está entre nosotros) y Rafael Alegre por su entusiasmo y apoyo logístico en el campo. A los evaluadores anónimos por sus comentarios y sugerencias. A Luis Borrero y Cecilia Pallo por la lectura y comentarios a una versión previa de este trabajo. A Karen Borrazzo y Laura Mendelzon por sus sugerencias. Por último, quiero agradecer a Nora Franco por la lectura de este manuscrito y por haberme dado la oportunidad de investigar en Patagonia durante ocho años.

\section{Referencias Citadas}

Aguerre, A.M. 2000. Las vidas de Pati en la Toldería Tehuelche del Río Pinturas y el Después. Facultad de Filosofía y Letras, Universidad de Buenos Aires, Buenos Aires.

Ainis, A.F., R.B. Guttenberg, R.L. Vellanoweth, J.M. Erlandson, W.E. Kendig, J. Colston y L. Thomas 2017. A cache within a cache: description of an Abalone "treasure-box" from the CASIN-14 Redwood Box Cache, San Nicolas Island, Alta California. California Archaeology 9 (1):79-105.

Amick, D.S. 2004. A possible ritual cache of great basin stemmed bifaces from the terminal Pleistocene-Early Holocene occupation of NW Nevada, USA. Lithic Tecnology 29 (2):119145.

Aschero, C. 1975. Ensayo para una clasificación morfológica de artefactos líticos aplicada a estudios tipológicos comparativos. Informe presentado al CONICET, Buenos Aires.

Aschero, C. 1983. Ensayo para una clasificación morfológica de artefactos líticos. Revisión del año 1975. Informe presentado al CONICET, Buenos Aires.

Barbería, E.M. 1995. Los Dueños de la Tierra en la Patagonia Austral, 1880-1920. Universidad Federal de la Patagonia Austral y Universidad Nacional de la Patagonia Austral, Río Gallegos.

Belardi, J.B., F. Carballo Marina, A. Nuevo Delaunay y H. De Angelis 2013. Raspadores de vidrio y de gres cerámico en la reserva tehuelche (Aonikenk) de Camusu Aike: aportes al conocimiento de las poblaciones indígenas de los siglos XIX y XX en el territorio de Santa Cruz. Relaciones de la Sociedad Argentina de Antropología XXXVIII (1):37-57.

Bement, L.C. 1991. The thunder valley burial cache: Group investment in a central Texas Sinkhole cementery. Plains Anthropology 36 (135):97-109.

Binford, L.R. 1978. Nunamiut Ethnoarchaeology. Academic Press, New York.

Binford, L.R. 1979. Organization and formation processes: Looking at curated technologies. Journal of Anthropological Research 35 (3):55- 273.

Binford, L.R. 1980. Willow smoke and dogs' tails: Hunter-gatherer settlement systems and archaeological site formation. American Antiquity 45 (1):4-20.

Borrero L.A. y H.D. Yacobaccio 1989. Etnoarqueología de asentamientos Aché. Cazadores-recolectores del Paraguay oriental. Journal de la Société des Américanistes 75:7-33.

Buscaglia, S. 2012. Poder y Dinámica Interétnica en la Colonia Española de Floridablanca. Una Perspectiva Histórica y Arqueológica (Patagonia, Argentina, Siglo XVIII). Editorial Académica Española, Saarbrücken.
Carballo Marina, F., J.B. Belardi, S. Espinosa y B. Ercolano 2000-2002. Tecnología y movilidad en el río Coyle, Santa Cruz. Cuadernos del Instituto Nacional de Antropología y Pensamiento Latinoamericano 19:89-107.

Casanueva, M.L., A. Castro Esnal y C. Pérez de Micou 2019. Indígenas, colonos y colonos indígenas. Arqueología de tiempos históricos y su abordaje: una experiencia en el SO de Chubut, Patagonia argentina. En Arqueología de la Patagonia: El Pasado en las Arenas, editado por J. Gómez Otero, A. Svoboda y A. Banegas, pp. 93-104. CONICET-IDEAUS, Buenos Aires.

Castro Esnal, A. 2014. Camino y Piedra. Rutas indígenas y Arqueología en la provincia de Chubut. Fundación de Historia Natural Félix de Azara, Buenos Aires.

Cirigliano, N.A. 2016. Movilidad de Grupos Indígenas y Aprovechamiento de Materias Primas entre el Extremo Sur del Macizo del Deseado y la Cuenca del Río Santa Cruz durante los Últimos 2.000 años (Provincia de Santa Cruz, Argentina). Tesis Doctoral inédita, Facultad de Filosofía y Letras, Universidad de Buenos Aires, Buenos Aires.

Cirigliano, N.A. y M.N. Vommaro 2014. Un asentamiento histórico en cercanías del cañadón Mercerá (Provincia de Santa Cruz, Argentina). En Entre Pasados y Presentes IV: Estudios Contemporáneos en Ciencias Antropológicas, editado por A. Castro Esnal, M. L. Funes, M. Grosso, N. Kuperszmit, A. Murgo y G. Romero, pp. 280-296. Asociación Amigos del Instituto Nacional de Antropología, Buenos Aires.

Erlandson, J.M., L. Thomas-Barnett, R.L. Vellanoweth, S.J. Schwartz y D.R. Muhs 2013. From the island of the Blue Dolphins: a unique nineteenth - century cache feature from San Nicolas island, California. The Journal of Island and Coastal Archaeology 8:66-78.

Fiore, D. y M. Ocampo 2009. Arte rupestre de la región margen norte del río Santa Cruz: una perspectiva distribucional. En Arqueología de Patagonia: Una Mirada desde el Último Confín Tomo 1, editado por M. Salemme, F. Santiago, M. Álvarez, E. Piana, M. Vázquez y M. Mansur, pp. 499-513. Editorial Utopías, Ushuaia.

Franco, N.V. 2002. Estrategias de Utilización de Recursos Líticos en la Cuenca Superior del Río Santa Cruz. Tesis Doctoral inédita, Facultad de Filosofía y Letras, Universidad de Buenos Aires, Buenos Aires.

Franco, N.V. 2008. La estructura tecnológica regional y la comprensión de la movilidad humana: tendencias para la cuenca del río Santa Cruz. En Arqueología del Extremo Sur del Continente Americano. Resultados de Nuevos Proyectos, editado por L.A. Borrero y N.V. Franco, pp. 119-154. IMHICIHU-CONICET, Buenos Aires. 
Franco, N.V. 2013. ¿Es posible diferenciar la existencia de grupos humanos con áreas de circulación distintas en el extremo sur de Patagonia durante el Holoceno tardío? En Tendencias Teórico Metodológicas y Casos de Estudio en la Arqueología de la Patagonia, compilado por A.F. Zangrando, R. Barberena, A. Gil, G. Neme, M. Giardina, L. Luna, C. Otaola, S. Paulides, L. Salgán y A. Tívoli, pp. 363-370. Altuna Impresores, Buenos Aires.

Franco, N.V., G. Brook, N.A. Cirigliano y A.L. Guarido 2013. Cronologías y paleoambiente en el cañadón Yaten Guajen (Provincia de Santa Cruz, Argentina). Libro de Resúmenes XVIII Congreso Nacional de Arqueología Argentina. Arqueología Argentina en el Bicentenario de la Asamblea General Constituyente del Año 1813, pp. 527-528. Universidad Nacional de La Rioja -CONICET, La Rioja.

Franco, N.V., A. Castro, N.A Cirigliano, M. Martucci y A Acevedo 2011. On cache recognition: an example from the area of the Chico river (Patagonia, Argentina). Lithic Technology 36 (1):37-52.

Franco, N.V. y N.A. Cirigliano 2009. Materias primas y movilidad humana entre las cuencas de los ríos Santa Cruz y Chico (Provincia de Santa Cruz, Argentina). Primeros resultados. En Arqueología de Patagonia: Una Mirada desde el Último Confín Tomo 1, compilado por M. Salemme, F. Santiago, M. Álvarez, E. Piana, M. Vazquez y M. Mansur, pp. 361-368. Editorial Utopías, Ushuaia.

Franco, N.V., N.A. Cirigliano, M. Ocampo, D. Fiore y A. Acevedo 2014. Las ocupaciones del Holoceno tardío en los cañadones basálticos del norte del rio Santa Cruz (Patagonia, Argentina). Intersecciones en Antropología 15:377-389.

Franco, N.V., N.A. Cirigliano, L. Vetrisano y P. Ambrústolo 2015 Raw material circulation at broad scales in southern Patagonia (Argentina): the cases of the Chico and Santa Cruz river basins. Quaternary International 375:72-83.

Franco, N.V., V. Cortegoso, L. Ferreyra, G. Fernando y V.A. Duran 2018. Human ranking of spaces and the role of caches: case studies from Patagonia (Argentina). Quaternary International 473:278-289.

Franco, N.V., A.L. Guarido, S. García Guráieb, M. Martucci y M. Ocampo 2010. Variabilidad en entierros humanos en la cuenca superior y media del río Santa Cruz (Patagonia, Argentina). En Arqueología Argentina en el Bicentenario de la Revolución de Mayo. XVII Congreso Nacional de Arqueología Argentina Tomo V, editado por J. Bárcena y H. Chiavazza, pp. 1901-1906. UNCuyo-CONICET, Mendoza.

Franco, N.V., C. Otaola y M. Cardillo 2007. Resultados de los trabajos exploratorios realizados en la margen norte del río Santa Cruz (Provincia de Santa Cruz, Argentina). En Arqueología de Fuego-Patagonia. Levantando Piedras, Desenterrando Huesos... y Develando Arcanos, editado por F. Morello, M. Martinic, A. Prieto y G. Bahamonde, pp. 541-553. Ediciones CEQUA. Punta Arenas.

Gómez Otero, J. 1987. Un raspador en vidrio confeccionado por una tehuelche meridional. Mundo Ameghiniano 7:1-3.

Gómez Otero, J. 1989-90. Cazadores tardíos en la zona fronteriza del paralelo 52 sur. I. El paraje de Juni Aike. Anales del Instituto de la Patagonia 19:47-71.

Gómez Otero, J. 1996-97. Rescate y reinserción de los mantos de pieles (quillangos) indígenas de Patagonia, en la Provincia del Chubut. Cuadernos del Instituto Nacional de Antropología y Pensamiento Latinoamericano 17:69-87.

Goñi, R.A. y P.E. Madrid 1998. Arqueología sin hornear: Sitios arqueológicos históricos y el Fuerte Blanca Grande. Intersecciones en Antropología 2:69-83.
Goñi, R.A. y A. Nuevo Delaunay 2009. La Arqueología como "fuente" de la Historia. En Arqueología de la Patagonia. Una Mirada desde el Último Confín Tomo 1, compilado por M. Salemme, F. Santiago, M. Álvarez, E. Piana, M. Vazquez y M Mansur, pp. 149-158. Editorial Utopías, Ushuaia.

Gradin, C.J. 1976. Parapetos de piedra y grabados rupestres de la meseta del lago Buenos Aires. Actas y Memorias del IV Congreso Nacional de Arqueología Argentina (Primera parte). Revista del Museo de Historia Natural de San Rafael II (1/4):315-337.

Gradin, C.J. 2000. Más Allá y Más Acá del Río Santa Cruz. Nuevo Offset, Buenos Aires.

Grange, R.T. 1964. A cache of scrapers near Crow Butte, Nebraska. Plains Anthropologist 9 (25):197-201.

Halvorsen, P. 2011. Identidades Enmascaradas en la Patagonia. Uniones entre Nativos y Foráneos. Patagonia Sur, Buenos Aires.

Hayden, B. y A. Cannon 1983. Where the garbage goes: Refuse disposal in the Maya Highlands. Journal of Anthropological Archaeology 2:117-163.

Hammer, Ø. 2017. PAST 3.15. Más información (http://folk. uio.no/ohammer/past/) (2 mayo 2019).

Imbelloni, J. 1949. Los Patagones. Características corporales y psicológicas de una población que agoniza. Runa II (1-2):5-58

Instituto Geográfico Nacional de la República Argentina 2018. Capas SIG. Más información (http://www.ign.gob.ar/ NuestrasActividades/InformacionGeoespacial/CapasSIG) (2 mayo 2019).

Jackson, D.S. 1991a. Raspadores de vidrio en Dinamarquero: reflejo de una encrucijada cultural. Anales del Instituto de la Patagonia 20:57-67.

Jackson, D.S. 1991b. Instrumentos de vidrio de Cuarto Chorrillo, costa de Bahía Santiago, Estrecho de Magallanes. Anales del Instituto de la Patagonia 20:69-74.

Jackson, D.S. 1999. Raspadores de vidrio en un asentamiento Aónikenk en el valle del zurdo, zona central de Magallanes. Anales del Instituto de la Patagonia 27:175-181.

Kendrick, G. 1968. The Mouth-Blown Bottle. Edwards Brothers, Ann Arbor.

Kornfeld, M., K. Akoshima, G. Frisom 1990. Stone tool caching on the North American plains: implications of the McKean site tool kit. Journal of Field Archaeology 17 (3):301-309.

Lindsey, B. 2020. Society for Historical Archaeology. Historic Glass Bottle Identification \& Information Website. (https://sha. org/bottle/body.htm) (3 enero 2020)

Lorrain, D. 1968. An archaeologist's guide to nineteenth century American glass. Historical Archaeology 2:35-44.

Martinic, M.B. y A.I. Prieto 1985-1986. Dinamarquero, encrucijada de Rutas indígenas. Anales del Instituto de la Patagonia 16:53-84.

Martinic, M.,A. Prieto y P. Cárdenas 1995. Hallazgo del asentamiento del jefe Aonikenk Mulato en el valle del Zurdo. Una prueba de sedentarización indígena en el periodo histórico final. Anales del Instituto de la Patagonia 23:87-94.

Martinic, M. y H. Roehrs 1991. Hallazgo de un asentamiento colonizador en la costa de Bahía Santiago (Estrecho de Magallanes). Evidencias de relación Pionera-Indígena. Anales del Instituto de la Patagonia 20:45-56.

Molina, M.J. 1969-70. El abrigo de los pescadores (Prov. Santa Cruz): informe preliminar sobre un corte estratigráfico practicado en 1965. Anales de Arqueología y Etnografía 1415:239-50. 
Moreno, F.P. 2007 [1879]. Exploración de la Patagonia Sur I. Por las Cuencas del Chubut y el Santa Cruz (1876-1877). Ediciones Continente, Buenos Aires.

Musters, G.C. 1911. Vida entre los patagones. En Universidad Nacional de la Plata Biblioteca Centenaria Tomo I, pp. 129388. Imprenta de Coni Hermanos, Buenos Aires.

Narborough, J. 2007 [1694]. El viajen de Sir John Narbrough al Mar del Sur. En Un Relato de Diversos Viajes y Descubrimientos Recientes, editado por R.C. Paredes, pp 73-165. Eudeba, Buenos Aires.

Nuevo Delaunay, A. 2007. Tecnología vítrea en el siglo XX, Lago Strobel (Santa Cruz, Argentina). En Arqueología de Fuego-Patagonia. Levantando Piedras, Desenterrando Huesos...y Develando Arcanos, editado por F. Morello, M. Martinic, A. Prieto y G. Bahamonde, pp. 853859. Ediciones CEQUA, Punta Arenas.

Nuevo Delaunay, A. 2012. Disarticulation of Aónikenk huntergatherer lifeways during the late nineteenth and early twentieth centuries: two case studies from Argentinean Patagonia. Historical Archaeology 46 (3):149-164.

Nuevo Delaunay, A., J.B. Belardi, F. Carballo Marina, M.J. Saletta y H. De Angelis 2017. Glass and stoneware knapped tools among hunter-gatherers in southern Patagonia and Tierra del Fuego. Antiquity 91 (359):1330-1343.

O'Connel, J.F. 1995. Ethnoarchaeology needs a general theory of behavior. Journal of Archaeological Research 3 (3):205-255.

Oliva, F., J. Moirano y M. Saghessi 1991. Estado de las investigaciones arqueológicas en el sitio Laguna de Puan 2. Boletin del Centro 2:127-138.
Oliva, G., L. González, P. Rial y E. Livrachi 2001. Áreas ecológicas de Santa Cruz y Tierra del Fuego. En Ganadería Ovina Sustentable en la Patagonia Austral. Tecnología de Manejo Extensivo, editado por P. Borrelli y G. Oliva, pp. 41-82. INTA, Río Gallegos.

Ortiz Castro, C. 2007. Botellas de Vidrio como Marcadores Sociales y Cronológicos, Siglos XVII-XX. Bases para un Catálogo Arqueológico de Colombia. Trabajo de Grado inédito presentado para optar por el título de Magíster en Antropología, Departamento de Antropología, Universidad de Los Andes, Bogotá.

Pallo, M.C. y K.B. Borrazzo 2017. The archaeology of contact in southern Patagonia: some issues to be resolved in the southwestern forest. Arts \& Humanities Open Access Journal 1 (4):135-138.

Panza, J.L., L.E Sacomani y J.C. Cobos 2005. Hoja Geológica 4969-III Laguna Grande. Provincia de Santa Cruz, Boletín $N^{\circ}$ 362. SEGEMAR, Buenos Aires.

Priegue, C.N. 2007. En Memoria de los Abuelos. Historia de Vida de Luisa Pascual, Tehuelche. Publitek, Bahía Blanca.

Rennie, P.J. y L.B. Davis 2016. The Clark Creek Cache in west central Montana, Lithic Technology, 41 (1):1-18.

Schiffer, M.B. 1972. Archaeological context and systemic context. American Antiquity 37 (2):156-165.

Sironi, O. 2010. Propuesta metodológica para el análisis descriptivo de vidrios "retocados" del noroeste de la provincia de Mendoza. $L a$ Zaranda de Ideas 6:129-143.

\section{Nota}

${ }^{1}$ Josefa Machado y Dora Machado, procedentes de la reserva tehuelche Camusu Aike, Provincia de Santa Cruz, Argentina (Gómez Otero 1987, 1996-97). 
